were due in part to a mathematical error, a congressional report has found.

Announced by President George W. Bush in 2003, the plant was to have captured and stored $90 \%$ of its carbon dioxide emissions. It was originally slated to cost $\$ 950$ million, but was cancelled in early 2008 when new estimates pegged its cost at $\$ 1.8$ billion (see Nature 451, 612-613; 2008).

But those estimates were not comparable, the Government Accountability Office report says. The \$950-million estimate was done in constant dollars; the \$1.8-billion figure included expected increases for inflation. The 2008 figure would have been $\$ 1.3$ billion if adjusted for constant dollars.

Steven Chu, the new energy secretary, said last week that he plans to meet soon with private partners involved in the project to discuss whether and how to take it forward.

\section{Lighter Higgs boson harder to find}

Physicists have placed fresh limits on the mass of the Higgs boson - the particle thought to confer mass on other matter.

The particle was assumed to have an energy (or equivalent mass) of between 114 and 185 gigaelectronvolts $(\mathrm{GeV})$. But, after analysing pooled data, researchers

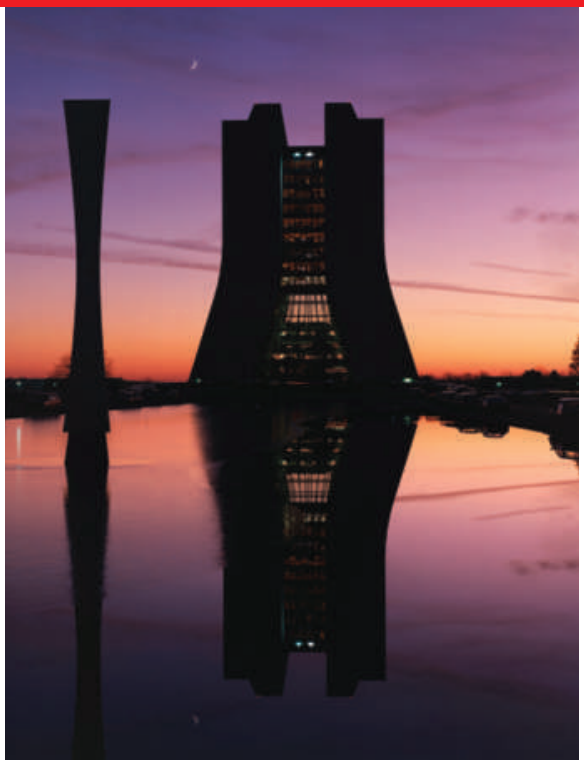

Fermilab's finding may cause problems for CERN.

at the Tevatron particle accelerator at the Fermi National Accelerator Laboratory in Batavia, Illinois, announced on 13 March that the particle can be excluded at energies of between 160 and $170 \mathrm{GeV}$, and is likely to exist at the lower end of the assumed range.

At more-crowded lower energies, filtering out other debris and finding a rare Higgs event becomes more difficult. Scientists at the Large Hadron Collider at CERN, Europe's particle-physics laboratory near
Geneva, had hoped to find the Higgs boson in the higher-energy region.

For a longer version of this story, see http://tinyurl.com/cp8avk.

\section{Hospital investigation reveals long-running fraud}

In what could be one of the largest ever cases of medical-research fraud, a prominent anaesthesiologist has been accused of fabricating data in at least 21 papers over 13 years.

Scott Reuben fabricated all or some of the data in studies as far back as 1996, according to an internal investigation by Baystate Medical Center in Springfield, Massachusetts, where he worked. Journals such as Anesthesiology and Anesthesia and Analgesia have already retracted Reuben's papers, which supported combining painkillers called COX-2 inhibitors (such as Merck's Vioxx and Pfizer's Celebrex) with other analgesics, including Pfizer's Lyrica, to relieve pain after surgery.

An attorney representing Reuben said that the Baystate investigation was confidential, that there were extenuating circumstances and that Reuben deeply regrets all that has happened. Reuben is barred from research at Baystate for at least a decade. 